050704

\title{
The Visual and the Haptic Müller-Lyer Illusions: Correlation Study
}

Edouard Gentaz, Valérie Camos, Yvette Hatwell and Annie-Yvonne Jacquet

\section{(2) OpenEdition}

\section{Journals}

Electronic version

URL: http://journals.openedition.org/cpl/431

DOI: $10.4000 / \mathrm{cpl} .431$

ISSN: $1379-6100$

Publisher

Centre PsyCLÉ

\section{Electronic reference}

Edouard Gentaz, Valérie Camos, Yvette Hatwell and Annie-Yvonne Jacquet, "The Visual and the Haptic Müller-Lyer Illusions: Correlation Study », Current psychology letters [Online], 13, Vol. 2, 2004 | 2004, Online since 05 July 2004, connection on 08 September 2020. URL : http:// journals.openedition.org/cpl/431; DOI : https://doi.org/10.4000/cpl.431

This text was automatically generated on 8 September 2020

(c) All rights reserved 


\section{4}

\section{The Visual and the Haptic Müller- Lyer Illusions: Correlation Study}

Edouard Gentaz, Valérie Camos, Yvette Hatwell and Annie-Yvonne Jacquet

\section{Introduction}

5 Studies on the haptic illusions corresponding to visual illusions are interesting from a theoretical point of view because they allow comparisons of the processes underlying these illusions (for reviews, see Hatwell, Streri, \& Gentaz, 2000, 2003; Heller, 2000; Millar, 1994). Thus, not finding a visual illusion in haptics is an argument in favour of specific haptic processes. However, observing the same illusion in vision and haptics does not allow us to conclude that the systematic perceptual error results from similar and/or specific processes. To answer this question, one possibility is to study whether the factors responsible for the presence and the variations of magnitude of the haptic illusion are identical to those affecting the same phenomenon in vision. An affirmative answer would bring arguments in favour of similar visual and haptic processes, whereas a negative answer would favour specific visual and haptic processes. The analysis of the literature about geometrical illusions (e.g., Müller-Lyer, VerticalHorizontal and Delboeuf illusions) in vision and haptics reveals that most of the above scenarios have been observed depending on the type of illusion studied (cf. Gentaz \& Hatwell, 2004). However, numerous studies provided evidence suggesting that the Müller-Lyer illusion may involve similar processes in both visual and haptic modalities.

In the Müller-Lyer illusion, the evaluation of the length of a segment is modified in independence of the orientation of the arrowheads situated at both ends. Thus, the segment with outward pointing arrowheads is overestimated as compared to the one (identical) with inward pointing arrowheads. Prior studies showed that the illusion is 
present in blindfolded sighted participants both visually and haptically (e.g., Heller et al., 2002; Lucca, Dellantonio, \& Riggio, 1986; Millar \& Al-Attar, 2002; Over, 1966a and b; Rudel \& Teuber, 1963; Suzuki \& Arashida, 1992; Wong, 1975). Some studies found similar magnitude of errors in both visual and haptic modalities whereas others observed a larger magnitude in vision (cf. Discussion).

In a number of studies, the presence of the Müller-Lyer illusion in vision and haptics seemed to result from similar visual and haptic processes because several factors affected the magnitude of the visual and haptic illusions in the same way. First, Rudel and Teuber (1963) found that the errors in the visual and haptic illusions decreased similarly as the number of trials increased although the participants received no feedback. Over (1967) confirmed this decrease in haptics. This suggests that the perceptual learning occurring very generally in vision is also at work in haptics. Second, Rudel and Teuber (1963) observed that this decrement in the magnitude of the illusion was equally transferable from haptics to vision and from vision to haptics. Third, the errors get stronger as the acute angles formed by the arrowheads and the segment to be evaluated get smaller in both vision (Coren \& Girgus, 1978) and haptics (Heller et al. 2002). Over (1966b) observed a similar effect of angle on the illusion magnitude in both modalities. Finally, Millar and Al-Attar (2002) showed that the same experimental manipulations reduced the Müller-Lyer illusion in vision and haptics to the same percentage error level. More precisely, detailed explicit instructions to ignore the arrowheads because they are confusing and to use body-centred cues for spatial reference reduced the Müller-Lyer illusion in both modalities to near zero. This illusion was not reduced in the absence of instructions to use body-centred cues, even when external reference cues were present. The authors suggested that an explicit egocentric reference may be the common factor underlying the Müller-Lyer illusion in both visual and haptic modalities.

8 If such a common factor exists, positive correlations between the visual and haptic errors of the participants should be observed. Curiously, this question has never been studied: Previous studies on the Müller-Lyer illusion in both modalities were always performed on independent groups of participants and therefore could not provide data on the intra-individual consistency of the illusion across modalities. However, the fact that this illusion is present in both modalities does not necessarily imply that the errors are correlated when they are measured in the same participants. For example, although the "oblique effect" (lower precision of the reproduction of oblique orientations by comparison to the reproduction of the vertical and horizontal orientations) is observed both in vision and haptics, no significant correlation between this visual and haptic oblique effect has been found when the same subjects were tested (Gentaz, Luyat, Cian, Hatwell, Barraud \& Raphel, 2001).

9 Therefore, to address this issue, we examined whether the Müller-Lyer illusion was correlated in visual and haptic tasks when the same blindfolded sighted adults performed exactly the same task in both modalities. Of course, the presence of a correlation will not demonstrate unambiguously that the same factor is responsible for the errors in the two modalities, but it could provide some support to this hypothesis. The same participants were asked to perform visually and then haptically (or in the reverse order) the task proposed by Rudel and Teuber (1963). The participants were asked to explore the display made of two arrowheads fixed at the end of rod which pointed to their left. Then, the participants were asked to give verbal instructions to 
the experimenter who moved the "central and third arrowhead" until they thought that its apex bisected the length of the rod. Thus, participants benefit from information only in the relevant modality. The counterbalanced order of presentation of the visual and haptic tasks raises a main question because half of the participants viewed the display before performing the haptic task, and we know from a number of previous studies that blindfolded sighted people tend to use their visual imagery when processing haptic tasks (cf. Hatwell, Streri and Gentaz, 2000, 2003). Therefore, in the analyses of results, this possible effect of order will be specially examined.

11 The first goal of our study was to verify that a classical Müller-Lyer illusion (perceptual error) was present in the visual and haptic modalities with the present experimental paradigm and to compare this illusion (if it is present) in the haptic and visual tasks. The second and main goal was to determine whether this illusion was correlated in the visual and haptic tasks. In addition, a third goal was to examine whether the response times were correlated in the visual and haptic tasks. Response time depends greatly on the duration of exploration of the display, and this duration may be related to perceptual error. Indeed, longer and systematic perceptual exploration may lead to more precise estimations because it allows a more complete analysis of the figure. Curiously, this question has never been studied in previous works. We know that, very generally, response time is longer in haptics than in vision. We were interested here not only in the absolute values of the response times in each modality, but also in their possible correlation with perceptual error in both modalities.

\section{Method2.1. Participants}

12 The participants were 30 right-handed adults in introductory psychology classes of René-Descartes University (Paris V). On the basis of self-reports, participants had no visual or somaesthetic disorders. They gave their informed consent.

2.2. Display, experimental conditions and procedure

13 The same display, similar to the one proposed by Rudel and Teuber (1963), was used for the two modalities. Two identical metal arrowheads (each side $40 \mathrm{~mm}$ in length) were fixed at the end of metal rod ( $5 \mathrm{~mm}$ thick and $150 \mathrm{~mm}$ long). The apex of these two arrowheads pointed to the participant's left (Figure 1). A third arrowhead, labelled the "central arrowhead", had exactly the same size and its apex pointed to the participant's right. It was rendered movable and could traverse the rod. The acute angle formed between each side of the arrowheads and the segment was 45 degrees. A digital sliding door (Fischer Darex) fixed behind the display was connected to the third arrowhead. It indicated in $\mathrm{mm}$ (the sensitivity threshold was $+/-0.03 \mathrm{~mm}$ ) the position of the third arrowhead along the rod. The display was positioned horizontally in the frontal plane and was centred on the participant's body midline. The height of the display could be varied so that it was adjusted at the level of the participant's eyes. The haptic and visual tasks were performed exactly in the same conditions.

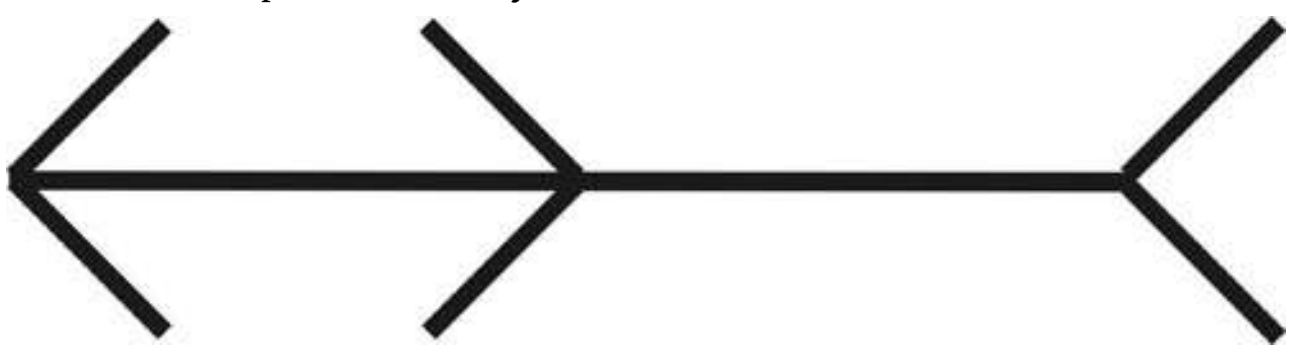

Figure 1: The display used in the visual and haptic tasks 
Each participant was tested individually and performed successively, in a counterbalanced order, the visual and the haptic tasks. Thus, half of participants started with the visual task then the haptic task, the other half performed these tasks in the reverse order. They were blindfolded when the task was haptic. The participants were asked to explore visually or haptically (with their right index finger, without visual control) the display and to give verbal instructions to the experimenter who moved the "central arrowhead" until they thought that its apex bisected the length of the rod. In both modalities, the starting position of the "central arrowhead" relative to the true middle was varied at each test trial. Two sides (right and left) and two lengths ( $55 \mathrm{~mm}$ and $65 \mathrm{~mm}$ ) were presented. In the visual task, the display was hidden by a screen between each test trial so that this starting position could be modified by the experimenter. In total, each participant performed 4 test trials in each starting position in a randomised order in both tasks (i.e., $4 \times 4 \times 2=32$ test trials per participant). In addition, a familiarisation phase was conducted before each modality task until the instructions were clearly understood (about 3 trials). In the haptic familiarisation phase, the experimenter guided the participant's right index finger over all parts of the display and asked the participant to explore the display in this way during the experimental phase. No time constraint was imposed in the presentation phase of the stimulus or the response phase. The duration of each task was about 20 min. A 5-min break was inserted between the tasks. The duration of the whole session was about 45 min for each participant. The total time, as well as the perceptual error, was measured for each test trial.

\subsection{Results}

The illusion (i.e. perceptual signed error) was measured by the difference between the estimated and actual middle, with positive value in $\mathrm{mm}$ indicating classical illusion (i.e. a deviation to the right of participant).

In order to determine whether a classical Müller-Lyer illusion was present in visual and haptic modalities, we compared the general mean of signed errors in each perceptual task to zero. The general visual errors $(M=8.6)$ and haptic errors $(M=6.4)$ were positive and $\mathrm{t}$ tests showed that they were significantly different from zero $(\underline{\mathrm{t}}(29)=$ $13.52, p<.0001$ and $t(29)=9.36, p<.0001$, respectively). These results confirmed the occurrence of the classical Müller-Lyer illusion in both modalities.

17 Second, to compare the perceptual errors and the response times, we conducted two 2 (order) x 2 (modality) ANOVAs with repeated measures on the last factor on mean signed errors in $\mathrm{mm}$ and on response times in seconds. The order had no effect on errors, $F(1,28)=1.15, p=.29$, or on times, $F(1,28)=3.75, p=.06$. The illusion was significantly smaller in the haptic $(\mathrm{M}=6.4 \mathrm{~mm} ; \mathrm{SD}=3.76)$ than in the visual task $(\mathrm{M}=8.6$

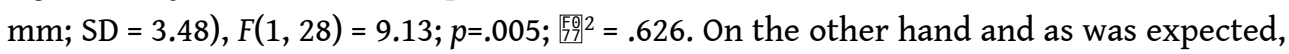
participants were significantly longer to give a response in the haptic task $(M=32.7 \mathrm{~s}$; $\mathrm{SD}=13.02)$ than in the visual task $(\mathrm{M}=14.3 \mathrm{~s} ; \mathrm{SD}=6.77), F(1,28)=96.77 ; p<.0001 ; \mathrm{Fig}^{2}=$. 872.

18 Third, we analysed the correlation between the visual illusion and the haptic illusion in the same participants. The mean signed errors (in $\mathrm{mm}$ ) of each participant in the visual and haptic tasks are reported in Figure 2. 


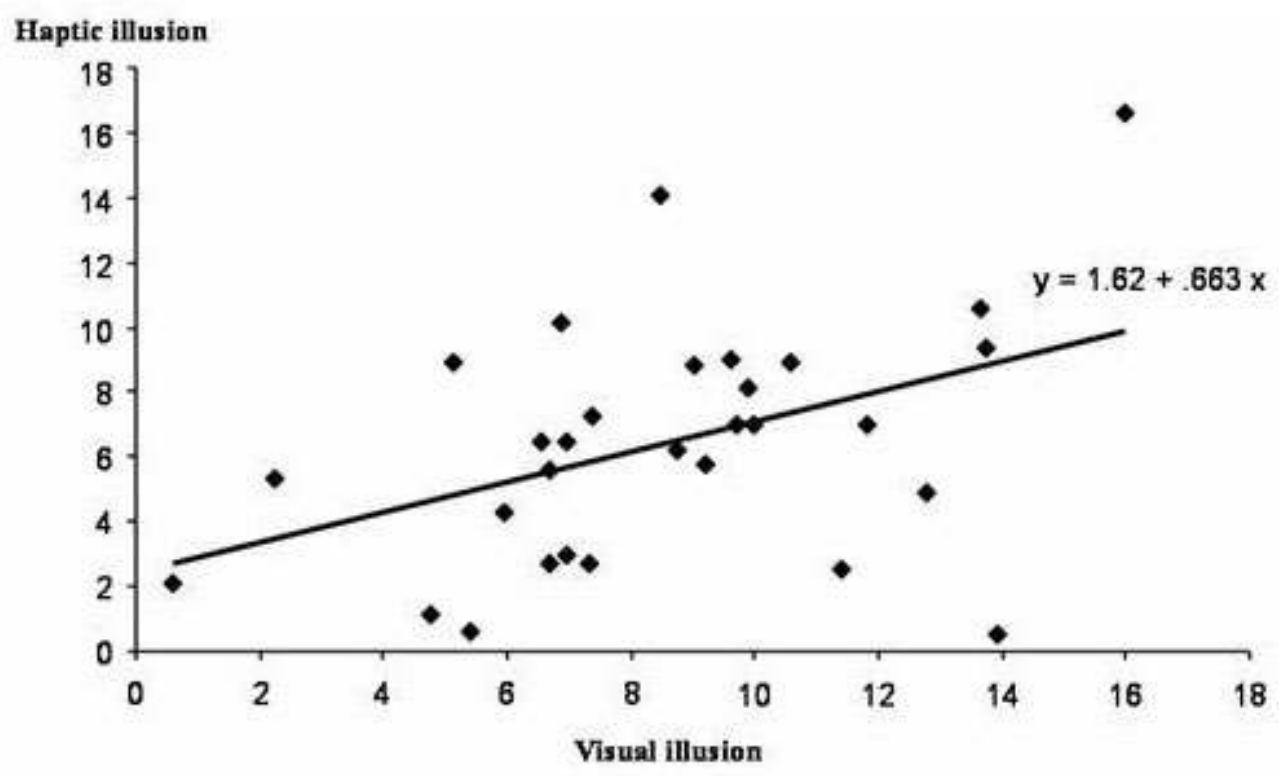

Figure 2: The haptic illusion in $\mathrm{mm}$ (mean signed errors) as a function of the visual illusion in each participant $(\mathrm{N}=30)$ and the regression line.

The analysis of the correlation on mean signed errors showed that the visual illusion was positively correlated with the haptic illusion, $r=0.62, F(1,28)=17.24, p<.001$. Moreover, similar analyses revealed that the response time for the visual illusion was also positively correlated to the time for the haptic illusion, $r=0.61, F(1,28)=16.87, p<$. 001.

Finally, the duration of exploration of the display did not seem to be related to perceptual error. The correlation between the response time and the perceptual error was not significant in vision $(r=.16, F<1)$ and in haptics $(r=.13, F<1)$.

3. Discussion

21 The results of present experiment confirmed the occurrence, with the display proposed by Rudel and Teuber (1963), of a classical Müller-Lyer illusion in the visual and haptic modalities. This illusion was higher in the visual task than in the haptic task. This result was consistent with Millar and Al-Attar's (2002, Experiment 4) study in which a classical display of two distinct stimuli was used. However, this result was not consistent with previous study which observed a similar illusion in both modalities with the same display. Indeed, with an identical stimulus (like in the present research), Rudel and Teuber (1963) obtained similar errors (about $3 \mathrm{~cm}$ ) in both modalities. It should be noted that this similarity was surprising because both the subjects and the mode of response (in vision, the experimenter moved the arrowhead whereas, in haptics, the participant moved it) were different in the two modalities. Thus, in the visual modality, participants benefit greatly from haptic information made available by the specific mode of response used. This suggests that the use of verbal instructions should be then favoured in any studies testing modality effects.

The most striking result is the positive correlation between the visual error and the haptic error in the same participants. This positive correlation was not affected by the order of presentation of the visual and haptic tasks. This means that the fact that half of the subjects viewed the display before performing the haptic task had no significant effect on the haptic error. Moreover, the response time for the visual task was also 
positively correlated to the time for the haptic one. These results are compatible with Millar and Al-Attar's hypothesis (2002) about the existence of a common process underlying the Müller-Lyer illusion in both visual and haptic modalities. The question remains to determine the nature of this possible common factor.

Millar and Al-Attar (2002) suggested that the explicit egocentric reference is this common factor. This hypothesis could account for our results. A complementary interpretation is that the observed correlations between the visual illusion and the haptic illusion may be partially explained by the visualisation process used by the participants during the haptic task. Indeed, most of the blindfolded sighted participants always use visual representations and visualisation when they have to use their hands to perceive the spatial properties of an object. However, other studies about the visual and haptic spatial perception did not find any correlation between the visual and haptic estimations. For example, and as stated in the introduction, this was the case in the perception of orientations and in the sensibility to the "oblique effect" (lower performance in oblique orientations than in vertical-horizontal ones). Although similar oblique effects were observed in both visual and haptic tasks (Luyat \& Gentaz, 2001, 2002), no positive correlations between the visual and haptic oblique effects were observed (Gentaz, et al. 2001). Further research should clarify the nature of the possible common processes underlying the visual and haptic Müller-Lyer illusions and the role of the visual sketchpad in the haptic illusion. Another explanation is that the common ability underlying the illusion in both modalities is the capability to inhibit the irrelevant information (i.e. the arrows) and to focus on goal-relevant information (i.e. the length of the rod). Engle (2002) suggested that this capability for controlling attention accounts for major individual differences in complex activities.

In conclusion, the existence of a positive correlation both between the visual and haptic errors and between the responses times of the visual and haptic tasks in the same observers is consistent with the hypothesis of non modality-specific common processes underlying the Müller-Lyer illusion in both modalities.

Acknowledgments

We thank Nathalie Schwalb and Laurent Warluzelle for technical support.

\section{BIBLIOGRAPHY}

Coren, S. \& Girgus, J. (1978). Seeing is deceiving: The psychology of visual illusions. Hillsdale, NJ: Lawrence Erlbaum Associates.

Engle, R. W.(2002). Working memory capacity as executive attention. Current Directions in Psychological Science, 11, 19 - 23 


\section{ABSTRACTS}

This study examines the Müller-Lyer illusion in the visual and haptic modalities. We investigated whether positive correlations between performances would be observed when the same participants performed exactly the same task in both modalities. The participants were asked to explore first visually only and then haptically only (or in the reverse order) a display made of two arrowheads fixed at the end of rod which pointed to their left. Then, the participants were asked to give verbal instructions to the experimenter who moved the "central and third arrowhead" until they thought that its apex bisected the length of the rod. Results revealed the presence of a significant positive correlation between the classical errors observed in the two modalities. Moreover, the response time for the visual task was also positively correlated to the time for the haptic one. The results are discussed in relation to the hypothesis assuming that common processes underlie the Müller-Lyer illusion in vision and haptics.

Cette recherche s'intéresse à l'illusion de Müller-Lyer dans les modalités visuelle et haptique (tactilo-kinesthésique). Nous avons examiné si les performances corrèlent quand les mêmes participants effectuent la même tâche dans les deux modalités. Les participants devaient explorer visuellement ou haptiquement un stimulus composé de deux pennures fixées au bout d'une baguette et orientées vers leur gauche et d'une troisième pennure mobile et orientée vers leur droite. Les participants donnaient des instructions verbales à l'expérimentateur de manière à ce qu'il place cette troisième pennure au milieu de la baguette. Les résultats révèlent une corrélation positive entre les classiques erreurs perceptives observées dans les deux modalités. De plus, les temps de réponse obtenus dans les deux tâches corrèlent aussi positivement. Les résultats apportent des arguments en faveur de l'hypothèse de processus communs responsables de l'illusion de Müller-Lyer dans les modalités visuelle et haptique.

INDEX

Keywords: perception, tactile, hand, vision, common process

\section{AUTHORS}

\section{EDOUARD GENTAZ}

Centre National of Scientific Research (CNRS), Laboratory "Cognition \& Development" (UMR 8605 CNRS), University René Descartes-Paris 5, 71 avenue Edouard Vaillant, 92774 Boulogne-Billancourt Cedex, France gentaz@psycho.univ-paris5.fr

\section{VALÉRIE CAMOS}

Centre National of Scientific Research (CNRS), Laboratory "Cognition \& Development" (UMR 8605 CNRS), University René Descartes-Paris 5, France

\section{YVETTE HATWELL}

Laboratory "Psychology \& NeuroCognition” (CNRS UMR 5105), University Pierre Mendès-France, Grenoble, France. 
ANNIE-YVONNE JACQUET

Centre National of Scientific Research (CNRS), Laboratory "Cognition \& Development" (UMR 8605 CNRS), University René Descartes-Paris 5, France. 